\title{
Full bandwidth coarse integral holographic video displays with spatial tiling for scalability
}

Li, Jin, Smithwick, Quinn, Chu, Daping

Jin Li, Quinn Smithwick, Daping Chu, "Full bandwidth coarse integral holographic video displays with spatial tiling for scalability," Proc. SPIE 11353, Optics, Photonics and Digital Technologies for Imaging Applications VI, 113530K (1 April 2020); doi: 10.1117/12.2556109

SPIE. Event: SPIE Photonics Europe, 2020, Online Only, France 


\title{
Full bandwidth Coarse Integral Holographic video displays with spatial tiling for scalability
}

\author{
Jin $\mathrm{Li}^{\text {a }}$, Quinn Smithwick ${ }^{\mathrm{b}}$, Daping Chu ${ }^{\mathrm{a}, *}$ \\ ${ }^{a}$ Centre for Photonic Devices and Sensors, Department of Engineering, University of Cambridge, \\ 9 JJ Thomson Avenue, Cambridge CB3 0FA, UK \\ ${ }^{\mathrm{b}}$ Disney Research, 521 Circle 7, Glendale, California 91201, USA \\ * Corresponding author: dpc31@cam.ac.uk
}

\begin{abstract}
This paper introduces full bandwidth Coarse Integral Holographic Display architecture with spatial tiling for scalability. The new method uses a large resonant scanner in conjunction with spatial tiling of spatial light modulators (SLMs) to create doubling the hologram's horizontal size and utilize the full bandwidth of the SLMs.
\end{abstract}

Keywords: Holography, Coarse Integral Holography, Spatial light modulators

\section{INTRODUCTION}

Holographic video displays are considered the frontier of 3D displays because it can generate arbitrary wavefronts that can deliver full 3D visual cues, hence it has great market potential for various applications in the future [1,2]. However, the display capabilities of current spatial light modulators (SLMs) cannot meet the requirements of the information content of a hologram with a large optical extent, i.e. the product of area and field-of-view (FOV). The main factor of the low display capabilities is that these SLMs have a low space bandwidth product (low-pitch and small area). Table 1 compares the optical extent of a hologram required for the display and a typically SLM. The comparison results demonstrate that the required optical extent is approximately 3881 times of the SLM pattern. An SLM with the pixel number of $1024 \times 1024$ and the pitches of $10 \mu \mathrm{m}$ couldn't directly display a large hologram (e.g. $127 \mathrm{~mm} \times 127 \mathrm{~mm}$ ) and wide FOV because the pitch should be less than $0.5 \mu \mathrm{m}$ for $\sim 30^{\circ} \mathrm{FOV}$. Thus, an efficient holographic display method is helpful to the improvement of the SLM display capability for the current holographic video with a large optical extent.

Table 1. The optical extent comparison of a hologram and a SLM display capability.

\begin{tabular}{|c|c|c|c|c|}
\hline Item & Pixel pitch & Size & FOV & Optical extent \\
\hline $\begin{array}{l}\text { Hologram } \\
\text { information }\end{array}$ & $\begin{array}{l}0.5 \mu \mathrm{m} \\
\text { (setting by the user) }\end{array}$ & $\begin{array}{l}60 \mathrm{~mm} \times 40 \mathrm{~mm} \\
\text { (setting by the user) }\end{array}$ & $\sim 30^{\circ}$ with $0.5 \mu \mathrm{m}$ & $\begin{array}{l}\sim 10^{6} \mathrm{~mm}^{2} \cdot \mathrm{deg}^{2} \text { (for } \\
60 \mathrm{~mm} \times 40 \mathrm{~mm} \text { and } 20^{\circ} \times 5^{\circ} \text { ) }\end{array}$ \\
\hline $\begin{array}{l}\text { SLM } \\
\text { capability }\end{array}$ & $\begin{array}{l}\text { the pitches of the SLMs } \\
\text { like DMD and LOCS is } \\
\text { only } 5 \sim 15 \mu \mathrm{m} \text { [3]-[4] }\end{array}$ & $\begin{array}{l}5 \mathrm{~mm} \sim 10 \mathrm{~mm} \\
\text { (for typical pixel numbers of } \\
1024 \times 1024 \text { ) }\end{array}$ & $1.17^{\circ}$ with $13 \mu \mathrm{m}$ & $\begin{array}{l}257.64 \mathrm{~mm}^{2} . \mathrm{deg}^{2} \\
\text { (for } 1024 \times 768 \text { pixels with } \\
\text { pixel pitch of } 13 \mu \mathrm{m} \text { ) }\end{array}$ \\
\hline
\end{tabular}

To adapt to the large optical extent generated by holograms, many 3D hologram display methods have been proposed to increase the size or FOV of the hologram. The combination of multiple SLMs using optical combing in conjunction with precision mounts in an arc has been demonstrated to increase the FOV [5]. A two-axis scanning mirror device has been used to time-sequentially tile reconstructed sub-holograms and achieve a large image size display of 50 Mpixel [6]. This approach has the potential to realise a gigapixel-level holographic display. The optical scanners have been used to construct a horizontally scanning holographic display system to increase the horizontal FOV [7]. A new acousto-optic SLM, in conjunction with a polygonal scanner, is used to implement the holographic video display system [8] to solve the low bandwidth and small diffraction angle of the traditional SLMs. Multiple physically tiled SLMs are used to implement a 3D holographic display with a large hologram size of 378 Mpixel and a video rate of $60 \mathrm{~Hz}[9]$.

We have developed a Coarse Integral Holography $(\mathrm{CIH})$ framework [10-14] to improve the SLM capability for 3D holographic video displays. Figure 1 shows the CIH frameworks. Current CIH displays can mainly be summarized into

Optics, Photonics and Digital Technologies for Imaging Applications VI, edited by

Peter Schelkens, Tomasz Kozacki, Proc. of SPIE Vol. 11353, 113530K · C 2020 SPIE

CCC code: $0277-786 X / 20 / \$ 21 \cdot$ doi: $10.1117 / 12.2556109$ 
two categories: $\mathrm{CIH}$ for a large FOV and $\mathrm{CIH}$ for large size. The $\mathrm{CIH}$ for large FOVs includes a static CIH method, a dynamic CIH method, and full bandwidth $\mathrm{CIH}$ method. The static and dynamic $\mathrm{CIH}$ methods use coarse integral optics and two galvanometer scanners to angularly tile several low-space-bandwidth-product (SBP) holograms, forming a modest size and wide field-of-view display. The full bandwidth CIH can be achieved using a resonant scanner and the combination of the multiple scanners to form the same size and double FOV display compared with the dynamic CIH. Under the framework of the full bandwidth CIH displays, the improvement of the CIH image size is developed. A full bandwidth $\mathrm{CIH}$ method using spatial tiling obtains the double increase of the horizontal holographic image size and the same full bandwidth FOV.

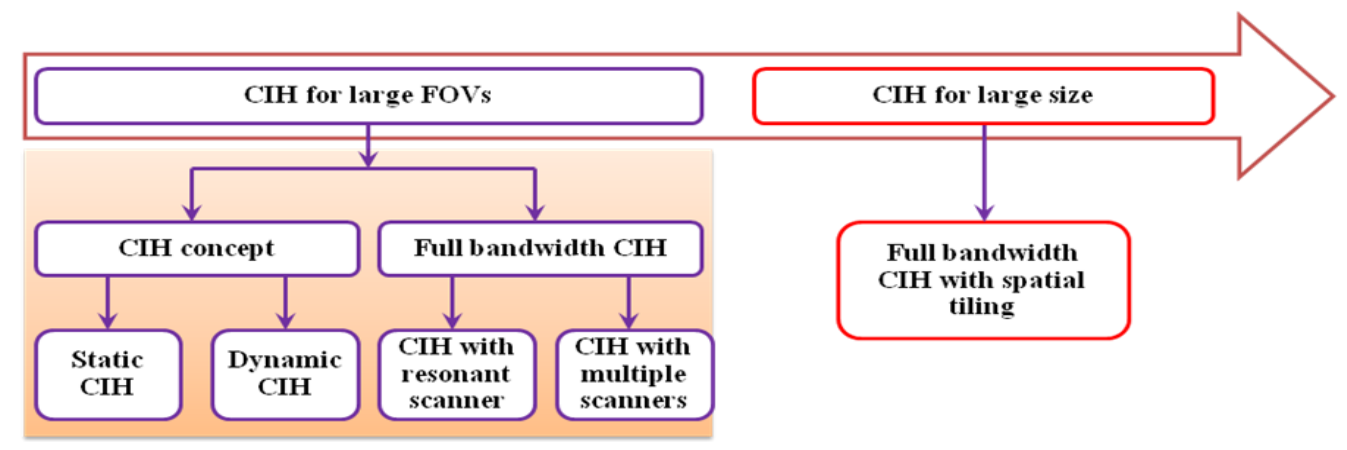

Figure 1. The CIH development framework involving two stages for large fields of view and large hologram size.

The remainder of the paper is organized as follows. Section 2 demonstrates the $\mathrm{CIH}$ display for large FOV, including static $\mathrm{CIH}$, dynamic $\mathrm{CIH}$, and full bandwidth $\mathrm{CIH}$. Section 3 presents the $\mathrm{CIH}$ display for large size, where the full bandwidth CIH with spatial tiling will be demonstrated. In Section 4, we conclude this paper.

\section{CIH FOR LARGE FIELD OF VIEWS}

In this section, we will present an overview of the CIH displays for a large FOV. In section 2.1, the principle of the static and dynamic CIH display is presented. Section 2.2 shows the full bandwidth CIH display method using a resonant scanner. In Section 2.3, three full bandwidth CIH methods using multiple scanners in the vertical or horizontal direction are introduced.

\subsection{Static and dynamic CIH}

The principle of the CIH display is that several low SBP holograms are angularly tiled using coarse integral optics to form a modest size and wide field-of-view display (i.e. larger optical extent). The coarse integral optics consists of a lens array and a large common transform lens. The array of low SBP holograms is attached behind the coarse integral optics and the lens array of the coarse integral optics matches with the hologram array. Each low SBP hologram with full 3D information represents a sub-view angle of the angular tiling. The arrangement of the low SBP hologram array can flexibly distribute and separately adjust view information between the horizontal and vertical FOV.

To demonstrate the $\mathrm{CIH}$ concept, both static and dynamic CIH displays have been implemented. Figure 2 shows the principle of the static and dynamic CIH displays. The concept-proof system of a static CIH display is achieved by a static array of holograms in a holographic film and coarse integral optics [10]. The static CIH utilizes coarse integral optics to efficiently combine multiple SLMs to increase the display's SBP. To decrease the number of the SLMs used in the static $\mathrm{CIH}$ display, the dynamic CIH display method was developed. It was achieved by angularly tiling multiple low SBP holograms for a larger image size and FOV using a high bandwidth SLM [11].

\subsection{Full bandwidth dynamic CIH method with a large resonant scanner}

At the current holographic video-rate speed in the dynamic CIH display, the scanning capability of the galvanometer mirror scanner limits the use of the SLMs bandwidth to only half of the SLM bandwidth. The angular tiling (without overlap) sub-holograms capability of the scanning system could not distribute all sub-holograms produced by the high bandwidth SLM at the current holographic video rate. Thus, the horizontal and vertical information content (i.e. the FOV) could not be fully utilized by this dynamic CIH system. 


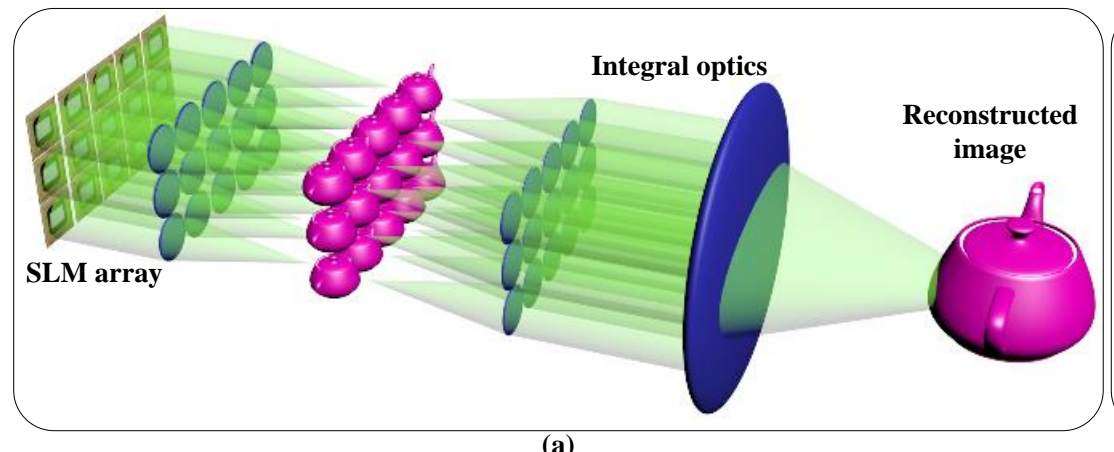

(a)

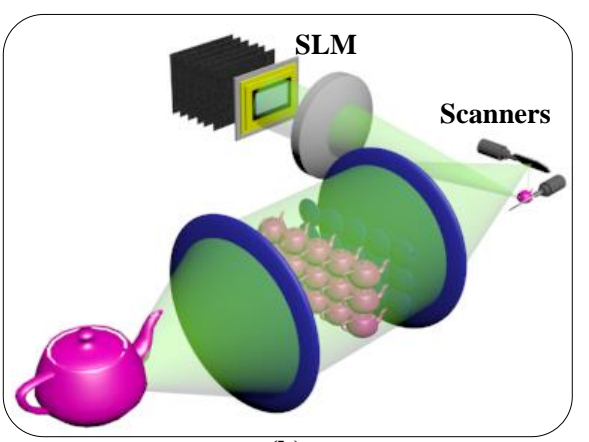

(b)

Figure 2. The principle of (a) the static $\mathrm{CIH}$ and the (b) dynamic CIH.

To fully utilize the SLM bandwidth of the dynamic CIH display, a full bandwidth CIH method using a large resonant scanner has been investigated [12]. We construct a 2D hybrid resonant scanning (HRS) system to replace the scanning system of the dynamic $\mathrm{CIH}$ system. Figure 3 shows the principle of the full bandwidth CIH displays with the HRS. The HRS is composed of a one-dimensional galvanometer scanner and a one-dimensional large resonant scanner in the vertical and horizontal, respectively. The large resonant scanner tiles low SBP sub-holograms produced by the high bandwidth SLM to form the horizontal FOV. The maximum scanning angle represents the maximum field of view angle (FOVA) tiled in the CIH system. In the full bandwidth CIH system with a large resonant scanner, the capability (denoted by $C$ ) of the proposed scanner system is calculated by $C=\beta \times F_{\text {scanner }} \times W_{\text {mirror }} \times H_{\text {mirror }}$, where $\beta$ is the deflection angle (field of view) of the scanner, $W_{\text {mirror }} \times H_{\text {mirror }}$ is the scanner mirror's area, $F_{\text {scanner }}$ is the scanning frequency. The deflection angle $\beta$ of a scanner is determined by the $F_{\text {scanner }}$, and $W_{\text {mirror }} \times H_{\text {mirror }}$. Let $\beta_{1}$ be the maximum deflection angle of the galvanometer scanner at the current maximum mirror size and the holographic video rate. We use $C_{1}$ to express the scanning capability of the original dynamic $\mathrm{CIH}$. Due to the resonant scanner's high scanning capability, it can achieve over twice the scan angle compared with the galvanometric scanner at the same frequency and mirror size. Thus, the maximum deflection angle resonant scanner is $\beta=2 \beta_{l}$. Accordingly, the scanning capability of the full bandwidth CIH scanning system is $C=2 C_{1}$. The twice scanning capability can distribute more extra holograms in each horizontal scan line, due to having longer scan lines shown in Fig.3 (b), to unutilized bandwidth of the SLM. Compared with the dynamic CIH, the full bandwidth CIH system can double the horizontal FOV and fully utilize the bandwidth of the SLM.

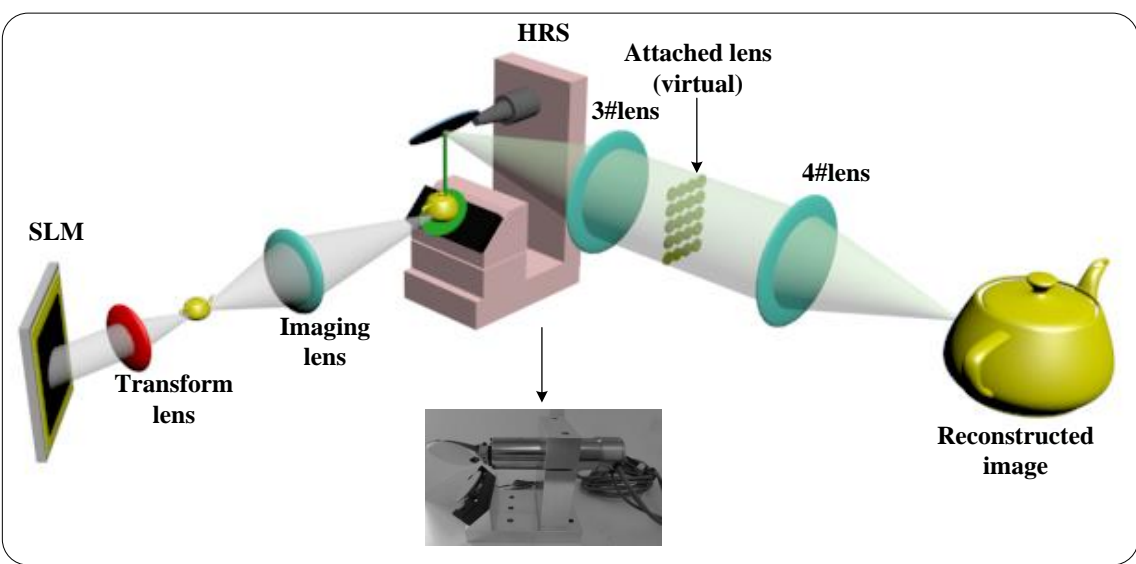

(a)

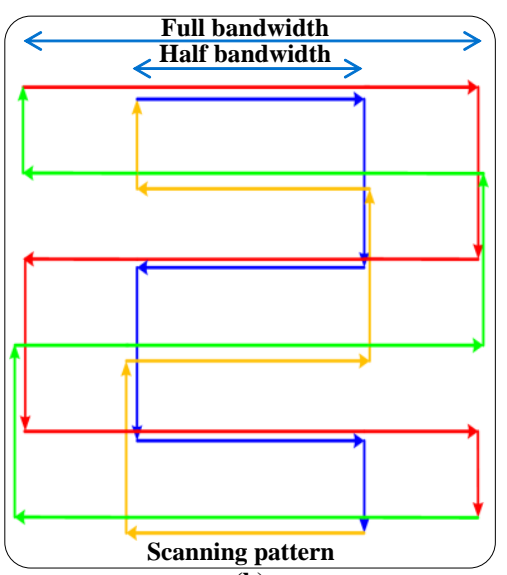

(b)

Figure 3. The principle of (a) the full bandwidth CIH using a resonant scanner and (b) achieving over twice the scan angle of the galvanometric scanner at the same frequency and mirror size.

\subsection{Full bandwidth dynamic CIH method with multiple scanners}

In the aforementioned dynamic $\mathrm{CIH}$, a vertical scanner and horizontal scanner are used in the vertical and the horizontal to allocate sub-holograms, respectively. We also have investigated the combination of multiple scanners in the vertical or horizontal direction to increase the optical extent distribution capability. In the vertical direction, we added an auxiliary high-speed resonant scanner into the original dynamic CIH system [13]. Figure 4 (a) shows the principle of the full 
bandwidth $\mathrm{CIH}$ using an auxiliary high-speed resonant scanner in the vertical direction. The combination of two vertical scanners can create vertical sub-lines for each horizontal scan line. Thus, the previously unused SLMs bandwidth in the display of sub-holograms is achieved by creating more vertical line resolution. Alternatively, we also used two cascaded synchronous scanners in tandem [14] to replace a horizontal scanner in the horizontal direction, while the vertical scanner keeps the same to the dynamic CIH. Figure 4 (b) shows the principle of full bandwidth $\mathrm{CIH}$ using two cascaded synchronous scanners in the horizontal direction. The combinations of two horizontal scanners can double the effective horizontal scan angle, obtaining twice the hologram's horizontal field of view. Thus, the full bandwidth of the SLM is utilized through tiling without overlap twice the previous number of sub-holograms using the horizontal multiple scanners.
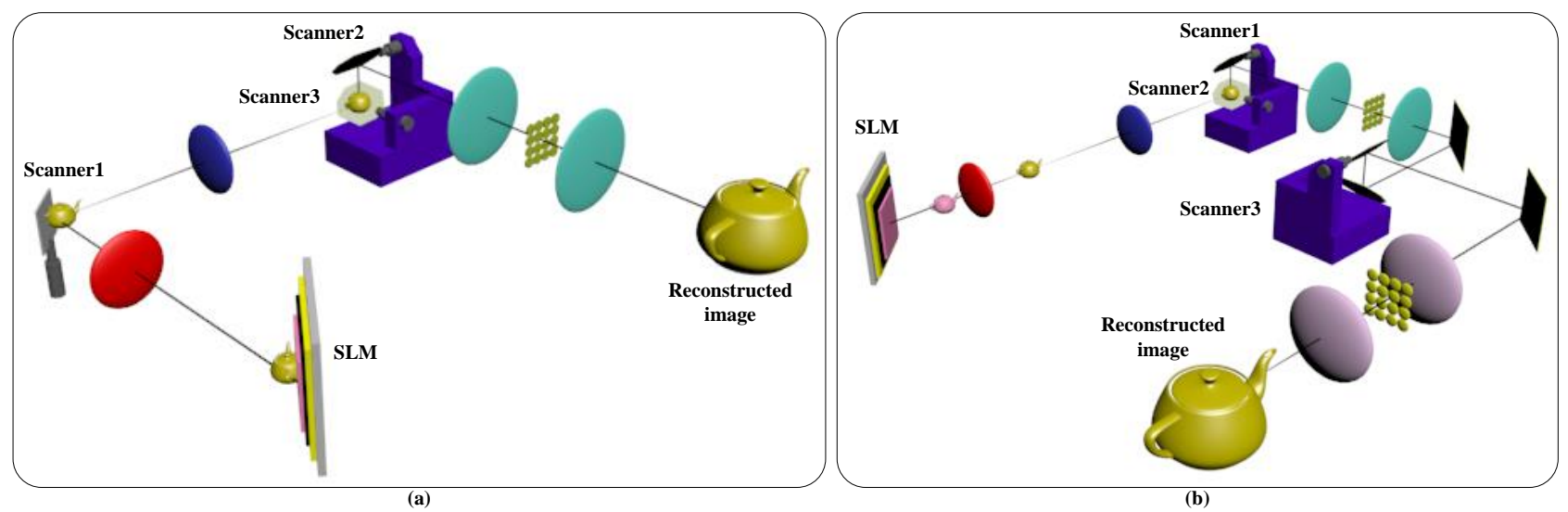

Figure 4. The principle of (a) the full bandwidth CIH using multiple scanners in the vertical direction or (b) horizontal direction.

\section{FULL BANDWIDTH CIH WITH SPATIAL TILING FOR SCALABILITY}

In this section, we will present the concept of the full bandwidth CIH with spatial tiling for scalability. In section 3.1, the principle of the spatial tiling method for $\mathrm{CIH}$ display is demonstrated. Section 3.2 compares different $\mathrm{CIH}$ display methods.

\subsection{The principle of the full bandwidth CIH with spatial tiling}

The SPB (representing hologram's information content) of a 3D holographic video can be calculated by $\mathrm{SBP}=S \times F O V \times F$, where $S$ is the hologram area size, $F O V$ is the total field of view in the horizontal and vertical direction, and $F$ is the display video frame rate. The full bandwidth methods (using a large resonant scanner or multiple scanners in the vertical or horizontal direction) allowed us to achieve the maximum FOV and fully use the SPB of the DMD. To further increase resolution, size or field of view of holographic displays, we need to add more information content (i.e. SPB) of a 3D holographic video into the full bandwidth CIH system through the additional SLMs. The two features should be followed when the $\mathrm{CIH}$ system adds more information content of a 3D holographic video. First, the CIH configurations (e.g. the scanners and optics) can accept, transform, and distribute the additional information (i.e. holographic image size) of light modulated by the additional SLMs. Second, the new CIH system is still scalable by adding additional SLMs, scanners, or optics to create the desired resolution, size, and FOV.

From these considerations, we demonstrate here a scalable $\mathrm{CIH}$ display with spatial tiling (stCIH). The stCIH uses spatially tiled multiple DMDs to replace the single DMD of the full bandwidth CIH display configuration (with a large resonant scanner) to increase holographic image size at the full bandwidth condition. Figure 5 shows the principle of the proposed CIH display with spatial tiling. The proposed stCIH display system constitutes three lasers, two SLMs, two Fourier transform lenses (L1 and L2), two imaging lenses (L3 and L4), a delay-mirror-array structure (DMAS), an HRS scanner, and a 4f-system (L5 and L6). The HRS composed by a one-dimensional resonant scanner (1D-RS) and a onedimensional galvanometric scanner (1D-GS) is the same as the full bandwidth CIH (with a large resonant scanner). The DMAS is used to remove the gap between seamlessly spatially tiled holographic images from two different DMDs. 1DRS is located on the image plane of the imaging lens (L3 and L4). The combined large holographic image is scanned by 
the 1D-RS and 1D-GS to form the horizontal and vertical FOV. The light direction vectors of two holographic images are completely parallel to still keep the integral image at different scanning views.

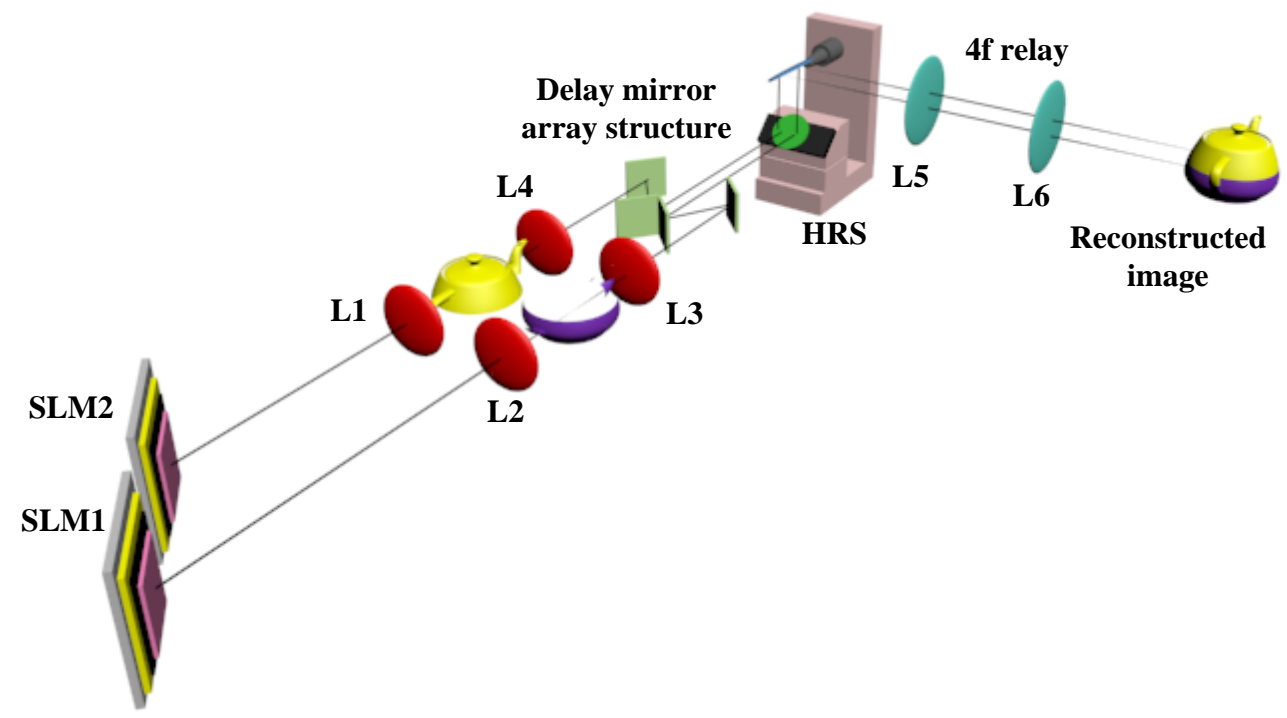

Figure 5. The principle of full bandwidth CIH using spatial tiling for enlarged hologram size.

\subsection{FOV and image size of full bandwidth CIH displays with spatial tiling}

Compared with the dynamic $\mathrm{CIH}$ system, the full bandwidth $\mathrm{CIH}$ system with spatial tiling obtained the advantages in the image size or FOV of the holographic displays. We will analyze the bandwidth, FOV, and image size for the stCIH in the following. The full bandwidth of an SLM can be calculated by $F B D=H \times V \times F$, where $H$ is the horizontal pixel resolution of the SLM, $V$ is the vertical pixel resolution of the SLM, and $F$ is the SLM's maximum pattern rate. In the dynamic CIH system, $H=1,024$ pixels, $V=768$ pixels, and $F=22,727 \mathrm{~Hz}$. Thus, the full SLM bandwidth of the dynamic $\mathrm{CIH}$ system is the $F B D=17.8 \times 10^{9} \mathrm{bit} / \mathrm{sec}$. In the dynamic $\mathrm{CIH}$, the vertical and horizontal galvanometer scanners adopt an efficient scanning method (i.e. boustrophedonic scan pattern shown in Fig.3) to create the horizontal and vertical FOV. The horizontal galvanometer scanner has a maximum frequency of $70 \mathrm{~Hz}$. To achieve the holographic video rate, the maximum scanning line number using the boustrophedonic scanning pattern is 6 . Thus, the holographic video frame rate of the dynamic $\mathrm{CIH}$ system is $23.33 \mathrm{~Hz}$. In the horizontal scanning line, we can layout 30 holograms tiled horizontally to form a horizontal angular FOVA of $24^{\circ}$. The vertical scanning line can layout 6 tiled holograms to form a vertical angular FOVA of $4.8^{\circ}$. Moreover, three lasers $(\mathrm{R} / \mathrm{G} / \mathrm{B}$ ) are integrated into the $\mathrm{CIH}$ system to obtain a color image (with three color components) of each sub-view to complete color holographic video displays. The used bandwidth of the SLM can be calculated by $U B D=H \times V \times V N \times S L \times N_{\text {laser }} \times B \times F_{D}$, where the $V N$ is the number of tiled views in each horizontal scanning line, the $S L$ is the number of scanning lines of each frame (i.e. the number of the tiled view in the vertical direction), $N_{\text {laser }}$ is the number of lasers (colors), $B$ is the bit depth of the SLM, and $F_{D}$ is the display video frame rate. In the dynamic CIH system with two galvanometer scanners, $H=1,024$ pixels, $V=768$ pixels, $V N=30$ views, $S L=6$ views, $N_{\text {laser }}=3$ colors, $B=1$ bit, and $F_{D}=23.33$ frame/s. Thus, the used bandwidth of the SLM in the dynamic CIH system is the $U B D=9.0 \times 10^{9} \mathrm{bit} / \mathrm{sec}$. The bandwidth utilization ratio is calculated by $R_{\text {bandwidth }}=U B D / F B D \times 100 \%$. Thus, the dynamic CIH display utilizes half of the SLM's full bandwidth. Using the same CIH configuration, the UBD is in proportion to the tile view number in the horizontal and vertical direction as

$$
U B D \propto V N \times S L \text {. }
$$

The total view of the display system is calculated by

$$
F O V=F O V_{x} \times F O V_{y}=\varphi_{x} \times V N \times \varphi_{y} \times S L
$$

where $\varphi_{x}$ is the horizontal FOV of each low SBP sub-hologram, $\varphi_{y}$ is the vertical FOV of a scanning line (i.e. vertical FOV of each low SBP sub-hologram). At the same CIH system, $\varphi_{x}$ and $\varphi_{y}$ depending on the diffraction angle are fixed. Combing Eq.1 and Eq.2, the relationship between the SLM's $U B D$ and the hologram's FOV is proportional as

$$
\mathrm{UBD} \propto \mathrm{FOV}_{x} \times \mathrm{FOV}_{y} .
$$


The stCIH system uses a large resonant scanner to scan the large tiled holographic images from two DMDs. Thus, the stCIH system is a full-bandwidth system. The full bandwidth CIH can provide the double number of views of each scan line compared with the original dynamic CIH method. Let $V N_{1}$ be the number of views of each scan line of the original dynamic CIH system. The number of views of each scan line in the full bandwidth becomes $V N^{\prime}=2 \times V N_{1}$. Let $U B D_{1}$ be the bandwidth of the original dynamic CIH system. Let $F O V_{1 x}$ be the horizontal FOV of the original dynamic CIH system. Based on Eq.1, the bandwidth utilization of the SLM becomes $U B D^{\prime}=2 \times U B D_{1}$. Based on Eq.3, the total horizontal field of view becomes $F O V_{1 x}=2 \times F O V_{1 x}$. As the displayed FOV in Fig. 6, two full bandwidth CIH methods can observe the holographic images with the FOV of $+20^{\circ} \sim 20^{\circ}$, while the half bandwidth $\mathrm{CIH}$ method is only $+10^{\circ} \sim 10^{\circ}$.

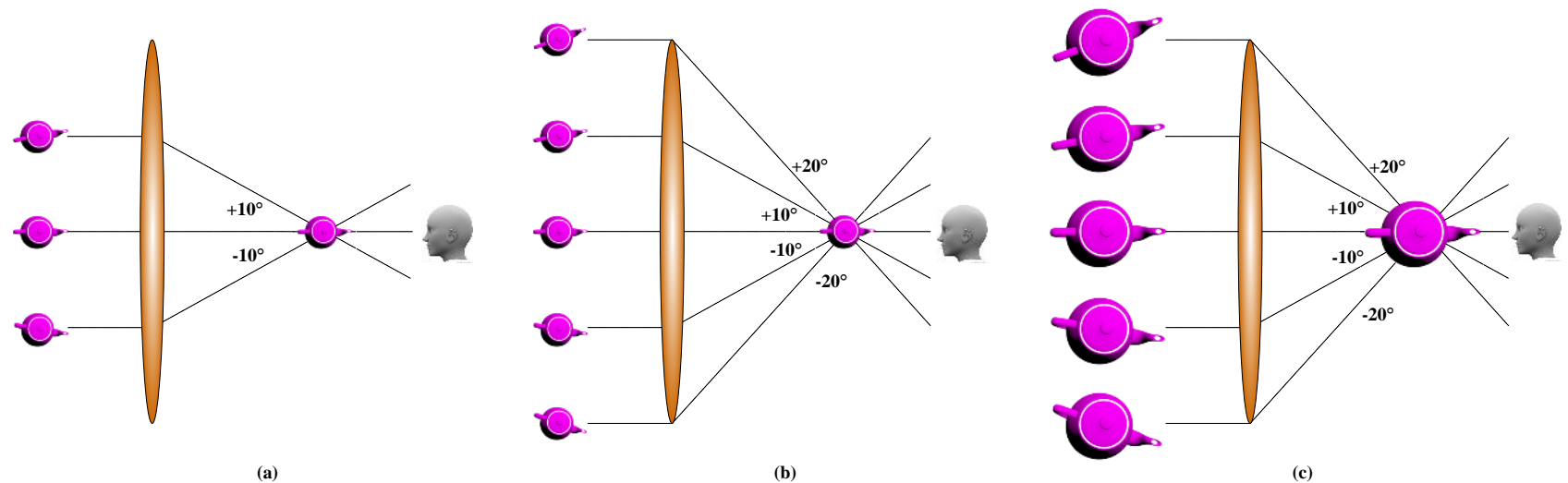

Figure 6. FOV and image size of the holographic information display using (a) half-bandwidth dynamic CIH, (b) fullbandwidth $\mathrm{CIH}$, and (c) spatial tiling full-bandwidth $\mathrm{CIH}$.

The holographic image size in the vertical or horizontal direction is determined by $L=M \Delta x=\mathrm{M} \times 2 \times f \times \tan (0.5 \times \theta)$, where $M$ is the pixel number of the hologram in the horizontal or vertical direction, $f$ is the diffraction distance, $\theta$ is the diffraction angle of the hologram. Let $M_{1}$ be the pixel number of the whole hologram of the full bandwidth CIH system with a large resonant scanner. We also use $L_{1}$ to express the holographic image size in the full bandwidth CIH system. In the stCIH system, the two SLMs are spatially tiled in the horizontal direction. Thus, the pixel number of the whole hologram of the stCIH system becomes $M^{\prime}=2 M_{1}$. Accordingly, the hologram size of the spatial tiling of two SLMs becomes $L^{\prime}=2 L_{1}$. In the stCIH system, large tiled holographic images with the size of $M^{\prime}$ is scanned by the common large resonant scanner and the common large scanning lens to form the horizontal FOV. As shown in Fig.6 (c), the holographic image size of the stCIH is the double of the full bandwidth CIH and the FOV keeps the same to the full bandwidth CIH. Table 2 shows other comparisons of different CIH displays.

Table 2. System comparisons of different CIH displays.

\begin{tabular}{|c|c|c|c|c|c|c|c|c|}
\hline Method & Colours & $\begin{array}{l}\text { No. of } \\
\text { DMDs }\end{array}$ & $\begin{array}{c}\text { DMD } \\
\text { bandwidth }\end{array}$ & FOV & $\begin{array}{c}\text { Hologram } \\
\text { size }\end{array}$ & $\begin{array}{c}\text { Frame } \\
\text { frequency }\end{array}$ & $\begin{array}{l}\text { Synchroni- } \\
\text { zation }\end{array}$ & Scalable \\
\hline Dynamic CIH & $\mathrm{R} / \mathrm{G} / \mathrm{B}$ & 1 & $53 \%$ & $\begin{array}{l}\text { Half of full } \\
\text { bandwidth FOV }\end{array}$ & $\begin{array}{l}\text { Without } \\
\text { improvement }\end{array}$ & $23.3 \mathrm{~Hz}$ & Easy & Easy \\
\hline $\begin{array}{l}\text { Full bandwidth } \\
\text { CIH }\end{array}$ & $\mathrm{R} / \mathrm{G} / \mathrm{B}$ & 1 & $\sim 100 \%$ & $\begin{array}{l}\text { Full bandwidth } \\
\text { FOV }\end{array}$ & $\begin{array}{l}\text { Without } \\
\text { improvement }\end{array}$ & $23.3 \mathrm{~Hz}$ & Moderate & Easy \\
\hline $\begin{array}{l}\text { Spatial tiling } \\
\text { CIH }\end{array}$ & $R / G / B$ & $\begin{array}{l}2 \text { (or } \\
\text { more) }\end{array}$ & $\sim 100 \%$ & $\begin{array}{l}\text { Full bandwidth } \\
\text { FOV }\end{array}$ & $\begin{array}{l}\text { Double } \\
\text { (or more) }\end{array}$ & $23.3 \mathrm{~Hz}$ & Moderate & Easy \\
\hline
\end{tabular}

\section{CONCLUSIONS}

To address the low display capability of current spatial light modulators for dynamic holographic videos, we developed a Coarse Integral Holography displays framework. First, we investigated a static CIH display and a dynamic CIH display method to improve the capability of the dynamic $\mathrm{CIH}$ to distribute hologram information. Second, three methods have been established to improve the bandwidth utilization, achieving a doubled horizontal field of view and full utilization of the SLM bandwidth. Third, we demonstrated full bandwidth Coarse Integral Holographic Display architecture with spatial tiling for scalability to double the hologram's horizontal size and still utilize the full bandwidth of multiple SLMs. In future work, we will develop large size CIH displays via tiling multiple systems to get a good field of view and image size in both directions. 


\section{REFERENCES}

[1] Häussler, R., Gritsai, Y., Zschau, E., Missbach, R., Sahm, H., Stock, M., \& Stolle, H., "Large real-time holographic 3D displays: enabling components and results," Applied Optics 56(13), F45-F52 (2017).

[2] Chen, J. S., \& Chu, D., "Realization of real-time interactive 3D image holographic display," Applied Optics 55(3), A127-A134 (2016).

[3] Jia, J., Chen, J., Yao, J., \& Chu, D. "A scalable diffraction-based scanning 3D colour video display as demonstrated by using tiled gratings and a vertical diffuser," Scientific Reports 7, 44656 (2017).

[4] Zhang, Z., You, Z., \& Chu, D. "Fundamentals of phase-only liquid crystal on silicon (LCOS) devices," Light: Science \& Applications 3(10), e213 (2014).

[5] Hahn, J., Kim, H., Lim, Y., Park, G., \& Lee, B., "Wide viewing angle dynamic holographic stereogram with a curved array of spatial light modulators," Optics Express 16(16), 12372-12386 (2008).

[6] Tanjung, R.B.A., Xu, X., Liang, X., Solanki, S., Pan, Y., Farbiz, F., Xu, B. and Chong, C.T., "Digital holographic three-dimensional display of 50-Mpixel holograms using a two-axis scanning mirror device," Optical Engineering 49(2), 025801 (2010).

[7] Takaki, Y. and Okada, N., "Reduction of image blurring of horizontally scanning holographic display," Optics Express 18(11), 11327-11334(2010).

[8] Smalley, D.E., Smithwick, Q.Y.J., Bove, V.M., Barabas, J. and Jolly, S., "Anisotropic leaky-mode modulator for holographic video displays," Nature 498(7454), 313-317 (2013).

[9] Xu, X., Liang, X., Pan, Y., Zheng, R., Lum, Z.A., Lwin, P.P.M.Y. and Solanki, S., "Development of full-color full-parallax digital 3D holographic display system and its prospects," In Practical Holography XXVII: Materials and Applications, International Society for Optics and Photonics, Vol. 8644, 864409 (2013).

[10] Smithwick, Q., Chen, J. S., Chu, D. “A Coarse Integral Holographic Display,” SID International Symposium 44(1), 310-313 (2013)

[11] J. S. Chen, Q. Y. J. Smithwick, and D. P. Chu, "Coarse integral holography approach for real 3D color video displays," Optics Express 24(6):6705-6718 (2016).

[12] Li, J., Smithwick, Q., \& Chu, D., "Full bandwidth dynamic coarse integral holographic displays with large field of view using a large resonant scanner and a galvanometer scanner," Optics Express 26(13), 1745917476 (2018).

[13] Chen, J.S., Smithwick, Q., Li, J. and Chu, D., "Auxiliary resonant scanner to increase the scanning capability of coarse integral holographic displays," Chinese Optics Letters 15(4), 040901 (2017).

[14] Li, J., Smithwick, Q., \& Chu, D., "Bandwidth utilization improvement methods of Coarse Integral Holographic video displays," In Digital Holography and Three-Dimensional Imaging, Optical Society of America, DTh3D-6 (2018). 